\title{
A Chinese Medicine Formula "Xian-Jia-Tang” for Treating Bladder Outlet Obstruction by Improving Urodynamics and Inhibiting Oxidative Stress through Potassium Channels
}

\author{
Jie Sun, ${ }^{1}$ Wei Shen, ${ }^{1}$ Wenjin An, ${ }^{1}$ Qiufen Li, ${ }^{2}$ Shunan Qiu, ${ }^{1}$ and Shaobo Jiang ${ }^{1}$ \\ ${ }^{1}$ Department of Urology, The First Affiliated Hospital of Zhejiang Chinese Medical University, Hangzhou City 310006, China \\ ${ }^{2}$ Department of Nephrology, Hangzhou Hospital of Traditional Chinese Medicine, Hangzhou City 310006, China \\ Correspondence should be addressed to Jie Sun; jiesun147@hotmail.com
}

Received 31 October 2016; Accepted 9 March 2017; Published 19 April 2017

Academic Editor: Giuseppe Morgia

Copyright (C) 2017 Jie Sun et al. This is an open access article distributed under the Creative Commons Attribution License, which permits unrestricted use, distribution, and reproduction in any medium, provided the original work is properly cited.

\begin{abstract}
The aim of this study is to investigate efficacy of a traditional Chinese medicine formula (named Xian-Jia-Tang, XJT) on bladder outlet obstruction (BOO) in rats and explore its mechanisms. Total $80 \mathrm{BOO}$ model rats were established and randomly divided into 4 groups: physiological saline, XJT, Cesium Chloride (CC), and XJT and CC groups. Meanwhile, 12 rats were used as normal control. Bladder weight and urodynamics were measured. Oxidative stress level and mRNA expressions of potassium channels gene were detected in detrusor. The mRNA and protein levels of hypoxia inducible factor- $\alpha$ (HIF- $\alpha$ ) in detrusor were detected by RTPCR and Western blot. BOO model rats showed significantly higher bladder weight and abnormal urodynamics. XJT significantly improved the abnormal urodynamics and inhibited the oxidative stress and changes of mRNA levels of potassium channels genes in detrusor of BOO model rats. Moreover, KATP and SK2/3 mRNA were overexpressed in BOO model rats treated by XJT. Besides, the significantly increased levels of HIF- $\alpha$ mRNA and protein were also inhibited by XJT. However, these inhibition effects of XJT were weakened by CC. XJT could effectively improve the urodynamics and inhibit the oxidative stress caused by hypoxia through suppressing the role of potassium channels in BOO model rats.
\end{abstract}

\section{Introduction}

Bladder outlet obstruction (BOO) is a common medical disorder in older males that refers to a blockage that slows or stops urine flow out of the bladder with marked alterations in bladder structure and function, which could result in various lower urinary tract symptoms (LUTS), such as weak urine stream, incomplete urination, frequent urination, and even urge incontinence [1]. $\mathrm{BOO}$ is the main consequence of benign prostate hyperplasia (BPH), which is a highly prevalent disease of older men caused by nonmalignant, unregulated growth of the prostate gland [2]. Currently, transurethral resection of the prostate is still the standard treatment of $\mathrm{BOO} / \mathrm{BPH}[3,4]$. However, there were many postoperative complications, such as urge incontinence and sexual dysfunction, which were associated with the decreased quality of life $[5,6]$. Thus, it is necessary to find the effective pharmacotherapy for the patients with BOO.
The $\alpha$-blockers and anticholinergic agents are the commonly used drugs for treatment of LUTS, BPH, and BOO at present $[7,8]$. Some studies have found the improved quality of life in $\mathrm{BOO}$ patients treated by these drugs $[9,10]$. However, the application of these drugs was limited by the side effects, such as dizziness, hypotension, and erectile dysfunction $[11,12]$. Therefore, new pharmacological strategies on the treatment of $\mathrm{BOO}$ are still needed.

Chinese medicine is always considered to be milder and does not have as strong side effects as Western drugs. XianJia-Tang (XJT) is a traditional Chinese medicine formula for invigorating kidney and promoting blood circulation (composition: root of Rehmannia glutinosa [shudi], leaves of Epimedium [yinyanghuo], fruit of barbary wolfberry [gouqizi], morinda root [bajitian], pangolin scales [chuanshanjia], root of Salvia miltiorrhiza [danshen], root of Rhizoma curcumae [eshu], and root of szechwan lovage rhizome [chuanxiong] at a rate of $2: 2: 3: 2: 1: 2: 1: 2$ in dry weight, 
including $2.34 \mathrm{~g}$ dry medicine/ml. XJT has been found to be able to suppress the proliferation of prostatic duct epithelium, reduce the deposition of collagen, and inhibit uncoordinated contraction of the bladder, thereby protecting the function of bladder in BPH rats [13]. The shudi, chuanshanjia, danshen, eshu, and chuanxiong can promote blood circulation, and yinyanghuo, gouqizi, and bajitian have an effect of strengthening kidney [13]; the functions of these compositions of XJT may be beneficial to the function of bladder. Many studies have indicated that the danshen and chuanxiong all could stimulate calcium-activated potassium channels in coronary artery smooth muscle cells of pigs and mesenteric artery smooth muscle cells of humans. Meanwhile, it was reported that the $\mathrm{BOO}$ was associated with the detrusor overactivity which was associated with the large-conductance calciumand voltage-activated potassium channels (BK) $[14,15]$. Thus, we speculated that the XJT also had efficacy in treating BOO. The mechanism may be associated with the activity of potassium channels in detrusor.

Thus, we performed this study to evaluate the effects of XJT on bladder weight and urodynamics in the BOO model rats. In addition, we also investigated the oxidative stress level and mRNA expression of potassium channels genes in detrusor to explore the mechanisms. The potassium channel inhibitor Cesium Chloride (CC) was used to confirm the role of potassium channels in the mechanism of XJT in treating BOO. In addition, a previous study showed that pharmacological inhibition of HIF pathways is beneficial for the bladder function in BOO model murine [16]. So, we also investigated the mRNA and protein expression of hypoxia inducible factor- $\alpha$ (HIF- $\alpha$ ) in detrusor of BOO model rats and evaluated the effect of XJT on its expression.

\section{Materials and Methods}

2.1. BOO Model and Treatment. A total of 108 male Wistar rats ( 3 months old) were used in this study. Among them, 12 rats were used as normal control and the rest 96 rats were used to establish the BOO model. Bladder outlet was partially obstructed. Simply, these rats were firstly anesthetized with phenobarbital. Abdominal cavity was opened by a midline incision to expose the urethrovesical junction. Then the bladder neck was loosely tied with a 2-0 silk thread to produce partial obstruction in bladder outlet. Finally, the incision was closed and penicillin $(0.1 \mathrm{mg} / \mathrm{kg})$ was given by intraperitoneal injection.

One week after surgery, 80 BOO model rats (83.3\%) survived. These $\mathrm{BOO}$ model rats were randomly divided into 4 groups with 20 rats in each group: in XJT group $(n=20)$, rats were given XJT ( $1 \mathrm{~mL} / 100 \mathrm{~g}$ body weight, each day) by gavage; in CC group, rats were intraperitoneal injected with nonradioactive CC (12 mg/100 g body weight, each day); in $\mathrm{XJT}+\mathrm{CC}$ group: rats were not only given XJT $(1 \mathrm{~mL} / 100 \mathrm{~g}$ body weight, each day) by gavage but also injected with CC (12 mg/100 g body weight, each day); and in BOO group, rats were given equal volume physiological saline by gavage. In addition, the 12 rats in normal control group were also treated with equal volume physiological saline. These treatments were continually performed for 30 days. This study was approved by Ethics Committee of the Local Animal Research.

2.2. Urodynamic Test. After 30 days of treatment, rats were anesthetized by phenobarbital, and the bladder was exposed by abdominal incision. Then epidural catheter was used as fistula by being inserted into the bladder and fixed with silk. This fistula was connected to a DISA System 2000 urodynamic instrument (Skovlunde, Denmark) and a CMA/100 microinjection pump (Stockholm, Sweden) via a T-tube. Finally, the maximum bladder capacity (MBC) and maximum detrusor pressure (MDP) were measured.

2.3. Tissue Sample Preparation. After urodynamic test, the rats were sacrificed by overdose of anesthetic drug. Afterwards, the bladders were rapidly removed and weighed. Then the whole bladders were kept in ice-cold Krebs salt solution until the detrusor was separated from the bladder mucosa by microdissection under sterile conditions. The halves of fresh detrusor muscles in each rat were immediately used for detecting some oxidative stress indices. The rest of detrusor muscles were stored in liquid nitrogen immediately and maintained at $-80^{\circ} \mathrm{C}$ until used for real-time polymerase chain reaction (RT-PCR) or Western blot.

2.4. Measurement of Oxidative Stress Indices. Malondialdehyde (MAD) was determined by the thiobarbituric acid assay in which a red-colored complex was produced through the reaction between MAD and thiobarbituric acid. This absorbance of this red-colored complex was measured at $532 \mathrm{~nm}$. The activity of superoxide dismutase (SOD) was measured by quantifying the inhibition of cytochrome-C reduction in xanthine-xanthine oxidase system at $550 \mathrm{~nm}$. The determination of total antioxidative capacity (T-AOC) was measured by using a commercial kit (Nanjing Jiancheng Bioengineering Institute, Nanjing, China) based on the instruction. A UV-2400PC spectrophotometer (Shimadzu, Kyoto, Japan) was used in these measurements.

2.5. Real-Time Polymerase Chain Reaction (RT-PCR). The frozen samples were thawed and homogenized at $4^{\circ} \mathrm{C}$ in $50 \mathrm{mM}$ Tris- $\mathrm{HCl}$ (pH 7.6), using a Teflon homogenizer. Total RNA was extracted using the Trizol reagent (Biobasic, Inc., Ontario, Canada). The cDNA was obtained by reversely transcribing these isolated RNA. Then RT-PCR was performed by a Bio-Rad iCycler (Bio-Rad, Hercules, CA, USA) to determine the mRNA levels of the following genes: Kv1.5 and Kv2.1 (encodes voltage-gated potassium channels), $B K$ (encodes large-conductance calcium-activated potassium channels), SK2/3 (encodes subtypes of small conductance calciumactivated potassium channels), and KATP (encodes activates adenosine triphosphate-sensitive potassium channels) as well as HIF- $\alpha$ (encodes hypoxia inducible factor- $\alpha$ ) by using the corresponding primers (Table 1). The reaction program included an initial denaturation at $95^{\circ} \mathrm{C}$ for $1 \mathrm{~min}$, followed by 40 cycles at $95^{\circ} \mathrm{C}$ for $10 \mathrm{~s}$ and $64^{\circ} \mathrm{C}$ for $25 \mathrm{~s}$. The mRNA levels of these genes were quantified using $18 \mathrm{~S}$ rRNA as internal control. 
TABLE 1: Special primers used in RT-PCR.

\begin{tabular}{|c|c|c|}
\hline Gene & Genbank accession & Primer sequences $\left(5^{\prime}\right.$ to $\left.3^{\prime}\right)$ \\
\hline \multirow{2}{*}{ Kv1.5 } & \multirow{2}{*}{ NM_012972.1 } & Forward: CTGGGGGTTCCCTGGAGAGTT \\
\hline & & Reverse: GCATACAGGGACCTCCGCAAGT \\
\hline \multirow{2}{*}{$K v 2.1$} & \multirow{2}{*}{ NM_013186.1 } & Forward: GAGGAGCACACGGGAGCACTA \\
\hline & & Reverse: GTGGTGGGGGCCTTGGAGTT \\
\hline \multirow{2}{*}{$B K$} & \multirow{2}{*}{ NM_031828.1 } & Forward: CGAGGGCCTCACTAAACCATC \\
\hline & & Reverse: GCCGAAGCCGAAAGCGAATA \\
\hline \multirow{2}{*}{ KATP } & \multirow{2}{*}{ NM_017023.1 } & Forward: GGACAACTGTGCTGGACCTGAAA \\
\hline & & Reverse: TCTCCACACAAGGAGTGCGGTT \\
\hline \multirow{2}{*}{$S K 2 / 3$} & \multirow{2}{*}{ NM_019315.2 } & Forward: AGGAGTACCAGCGCCAGGTGT \\
\hline & & Reverse: CGGTGAATGGTGCCCGTGA \\
\hline \multirow{2}{*}{$H I F-1$} & \multirow{2}{*}{ Y09507 } & Forward: TGCCCGAAAGCTCGAACTCA \\
\hline & & Reverse: GACTTTGGCGTGGTCAATCTTCTT \\
\hline \multirow{2}{*}{$18 S$} & \multirow{2}{*}{ NR_046237 } & Forward: CGTGCCCCTACTATGTCGCTTT \\
\hline & & Reverse: GTCTTCTGCTCCATTCCATCCTGT \\
\hline
\end{tabular}

2.6. Western Blot. Western blot was performed to detect the expression of HIF- $\alpha$ in detrusor. Simply, the frozen samples were thawed and homogenized at $4^{\circ} \mathrm{C}$ in $50 \mathrm{mM}$ Tris$\mathrm{HCl}$ ( $\mathrm{pH}$ 7.6), using a Teflon homogenizer. Total proteins were extracted by RIPA Lysis Buffer (Beyotime Institute of Biotechnology, Shanghai) with protease inhibitor cocktail (Sigma, USA). These proteins were separated by sodium dodecyl sulfate-polyacrylamide gel electrophoresis with $10 \%$ polyacrylamide gels. Then the proteins were transferred to a polyvinylidene fluoride membrane (Millipore, USA) and blocked with $5 \%$ skim milk in Tris buffered saline Tween (10 mm Tris, $15 \mathrm{~mm} \mathrm{NaCl}, 0.05 \%$ Tween 20) for $2 \mathrm{~h}$. After incubation with primary antibody (anti-HIF-1, 1:500, sc10790, Santa Cruz) overnight at $4^{\circ} \mathrm{C}$, horseradish peroxidaselabeled second antibody $(1: 2000$, Santa Cruz) was added and continually incubated for $1 \mathrm{~h}$ at $25^{\circ} \mathrm{C}$. Finally, the proteins were visualized using enhanced chemiluminescence reagents (Amersham Pharmacia, UK). The density of protein bands was analyzed by Bandscan 5.0 software (Glyko, Novato, CA, USA). The relative level of HIF- $\alpha$ was calculated using $\beta$-actin as internal control.

2.7. Statistical Analyses. All data were expressed as mean \pm SD. Statistical analysis was performed by SPSS version 17.0 (SPSS Inc., Chicago, IL). Comparison among groups was determined by one-way analysis of variance using least significant difference test (equal variances assumed) or Dunnett's T3 (equal variances not assumed) for post hoc test between groups. A $P$ value less than 0.05 was considered as statistically significant.

\section{Results}

3.1. Changes of Bladder Weight. As shown in Table 2, the BOO model rats showed significantly lower body weight but higher bladder weight compared with the normal rats $(P<0.01)$. Moreover, the ratio of bladder weight/body weight was significantly higher in the rats of $\mathrm{BOO}$ group compared with that in the rats of normal control group $(P<$
0.01). However, the rats in XJT group indicated significantly higher body weight and lower bladder weight and ratio of bladder weight/body weight compared with that in $\mathrm{BOO}$ group $(P<0.01)$. Although the bladder weight and ratio of bladder weight/body weight in rats treated by XJT were still significantly higher compared with normal value $(P<0.01)$, the similar body weight was found between XJT and normal control groups $(P>0.05)$. After being injected with $C C$, the BOO model rats indicated significantly lower body weight and higher ratio of bladder weight/body weight $(P<0.01)$. XJT did not significantly inhibit the decreased body weight of BOO model rats that were injected with $\mathrm{CC}(P>0.05)$, while ratio of bladder weight/body weight was significantly decreased in rats of XJT + CC group. The bladder weight was similar among the rats in BOO, CC, and XJT + CC groups $(P>0.05)$. Moreover, the body weight was significantly decreased and bladder weight was significantly increased by CC in XJT + CC group when compared with that in XJT group $(P<0.01)$.

3.2. Effect of XJT on Urodynamics of BOO Model Rats. The results showed that the rats in $\mathrm{BOO}$ group had significantly higher MDP and lower MBC $(P<0.01)$, indicating urodynamic abnormality. XJT significantly decreased the MDP $(P<0.01)$ and increased the MBC of BOO model rats $(P=0.03)$, which was similar to the value in normal control group $(P>0.05)$. In addition, the significantly higher MDP was also found in the rats of CC group $(P<0.01)$, which was similar to that in the rats of $\mathrm{BOO}$ group $(P>0.05)$. Moreover, the mean level of $\mathrm{MBC}$ in $\mathrm{CC}$ group was significantly lower than that in normal control group $(P<0.01)$ and $\mathrm{BOO}$ group $(P=0.02)$. However, XJT could significantly inhibit the increase of MDP $(P<0.01)$ and the decrease of MBC $(P=0.03)$ in XJT + CC group. Moreover, compared with XJT group, CC significantly inhibited the effect of XJT on MDP $(P=0.04)$ and $\mathrm{MBC}(P<0.01)$ in XJT + CC group (Table 3$)$.

3.3. Effect of XJT on Oxidative Stress in Detrusor. BOO model rats exhibited significantly downregulated level of T-AOC 
TABLE 2: Bladder weight differences of rats in each group.

\begin{tabular}{|c|c|c|c|}
\hline Groups & Body weight (g) & Bladder weight (g) & Bladder weight/body weight (\%o) \\
\hline Normal control group & $326.25 \pm 16.02$ & $0.10 \pm 0.01$ & $0.31 \pm 0.03$ \\
\hline BOO group & $303.05 \pm 13.14^{* *}$ & $0.43 \pm 0.05^{* *}$ & $1.43 \pm 0.17^{* *}$ \\
\hline XJT group & $320.57 \pm 35.16^{\# \#}$ & $0.33 \pm 0.10^{* * \# \#}$ & $1.01 \pm 0.30^{* * \# \#}$ \\
\hline CC group & $265.40 \pm 21.81^{* * \# \# \Delta \triangle}$ & $0.47 \pm 0.08^{* * \Delta \Delta}$ & $1.77 \pm 0.03^{* * \# \# \triangle \triangle}$ \\
\hline XJT + CC group & $266.30 \pm 10.29^{* * \# \# \Delta \Delta}$ & $0.41 \pm 0.02^{* * \Delta \Delta}$ & $1.14 \pm 0.57^{* * \& \&}$ \\
\hline
\end{tabular}

BOO: bladder outlet obstruction; XJT: Xian-Jia-Tang (a traditional Chinese medicine formula for invigorating kidney and promoting blood circulation); and CC: Cesium Chloride. $* *, \#, \triangle \triangle$, and \&\&, respectively, represent significant difference compared with Normal control, BOO, XJT, and CC groups with $P<$ 0.01 .

TABLE 3: Changes of urodynamic parameters in the rats of each group.

\begin{tabular}{lcc}
\hline Groups & $\mathrm{MDP}\left(\mathrm{mmH}_{2} \mathrm{O}\right)$ & $\mathrm{MBC}(\mathrm{ml})$ \\
\hline Normal control group & $106.10 \pm 6.91$ & $3.00 \pm 0.34$ \\
BOO group & $160.33 \pm 10.37^{* *}$ & $2.35 \pm 0.29^{* *}$ \\
XJT group & $110.29 \pm 10.86^{\# \#}$ & $2.78 \pm 0.30^{\#}$ \\
CC group & $156.83 \pm 18.87^{* * \Delta \Delta}$ & $1.87 \pm 0.29^{* * \# \Delta \Delta}$ \\
XJT + CC group & $129.88 \pm 11.60^{* * \# \# \& \&}$ & $2.29 \pm 0.54^{* * \Delta \Delta \&}$ \\
\hline
\end{tabular}

BOO: bladder outlet obstruction; XJT: Xian-Jia-Tang (a traditional Chinese medicine formula for invigorating kidney and promoting blood circulation); CC: Cesium Chloride; MBC: maximum bladder capacity; and MDP: maximum detrusor pressure. $\#, \triangle$, and \&, respectively, represent significant difference compared with BOO, XJT, and CC groups with $P<0.05 ; * *$, \#\#, $\triangle \triangle$, and \&\&, respectively, represent significant difference compared with Normal control, BOO, XJT, and CC groups with $P<0.01$.

$(P=0.01)$ and upregulated levels of MAD and SOD $(P<$ 0.01 ) when compared with the rats in normal control group. By the intervention of XTJ, the levels of MAD, SOD, and TAOC were all recovered to normal level $(P>0.05)$. Similar to the BOO group, the levels of MAD $(P<0.01)$ and SOD $(P=0.02)$ were also significantly increased in CC group compared with those in normal control group. Moreover, XJT significantly reduced this increased level of MAD $(P<0.01)$ but did not affect the level of SOD $(P>0.05)$ in XJT + CC group, compared with that in CC group. In addition, results only showed a decreased trend of level of T-AOC in CC group without statistically significant difference $(P>0.05)$ compared with that in normal control group, which was also similar to that in BOO group $(P>0.05)$. Meanwhile, no significantly different levels of T-AOC were found between $\mathrm{CC}$ and XJT + CC group $(P>0.05)$. Besides, results also showed the inhibition effects of XJT on the levels of MAD $(P<0.01)$ and SOD $(P=0.03)$ were significantly weakened by CC when comparing XJT + CC group with XJT group (Figure 1).

3.4. Effect of XJT on Potassium Channels in Detrusor. As shown in Table 4, the mRNA expressions of $B K, S K 2 / 3$, and $K A T P$ were significantly reduced $(P<0.01)$, while the mRNA expressions of $K v 2.1$ and $K v 1.5$ were significantly increased $(P<0.01)$ in BOO model rats when compared with normal rats. However, XJT significantly increased the mRNA levels of $B K, S K 2 / 3$, and $K A T P(P<0.01)$ and decreased the mRNA levels of $K v 2.1(P<0.01)$ and $K v 1.5(P=0.04)$ in the rats of
XJT group compared with those in the rats of BOO group. The level of $B K$ mRNA was still lower and the levels of $K v 2.1$ and $K v 1.5$ mRNA were still higher in XJT group compared with the normal control group $(P<0.01)$, while the levels of $S K 2 / 3$ and KATP mRNA in XJT even exceeded the normal values $(P<0.01)$. The injection of CC did not significantly affect the levels of $B K, S K 2 / 3$, and $K v 1.5$ mRNA $(P>0.05)$ but significantly decreased the mRNA level of $K v 2.1(P=0.02)$ and increased the mRNA of $\operatorname{KATP}(P<0.01)$ in BOO model rats. However, XJT significantly decreased the levels of $K v 2.1$ and $K v 1.5$ mRNA $(P<0.05)$ and increased the level of KATP mRNA in XJT + CC group compared with that in CC group $(P<0.01)$ but did not significantly affect the levels of $B K$ and SK2/3 mRNA $(P>0.05)$. Moreover, compared with XJT group, CC significantly inhibited the effect of XJT on the mRNA levels of $B K, S K 2 / 3, K v 2.1$, and $K A T P$ in XJT + CC group $(P<0.05)$.

3.5. Effect of XJT on Expression of HIF- $\alpha m R N A$ and Protein in Detrusor. As shown in Figure 2, the mRNA and protein levels of HIF- $\alpha$ were significantly increased in BOO model rats compared with those in normal rats $(P<0.01)$. However, the upregulated expressions of HIF- $\alpha$ mRNA and protein in BOO model rats were significantly reduced by XJT $(P<$ 0.01 ). Although the level of HIF- $\alpha$ mRNA in XJT group was still higher than that in normal control group $(P<0.01)$, the level of HIF- $\alpha$ protein was decreased to a lower value by XJT $(P<0.01)$. The levels of HIF- $\alpha$ mRNA and protein in CC group were similar to those in BOO group $(P>0.05)$, which were also significantly higher than those in normal control group $(P<0.01)$. In XJT + CC group, XJT did not significantly decrease the higher levels of HIF- $\alpha$ mRNA and protein in the $\mathrm{BOO}$ model rats that were injected with $\mathrm{CC}$ $(P>0.05)$. However, CC significantly affected the inhibition effect of XJT on expression of HIF- $\alpha$ mRNA and protein in $\mathrm{XJT}+\mathrm{CC}$ group when compared with that in XJT group $(P<0.01)$.

\section{Discussion}

In this study, XJT, as a traditional Chinese medicine formula for treatment of BOO without strong side effects as Western drugs, was investigated in rats. Results showed that XJT could significantly inhibit the increased bladder weight and abnormal changes in urodynamics, indicating the role of $\mathrm{XJT}$ in preventing bladder damage and protecting bladder 


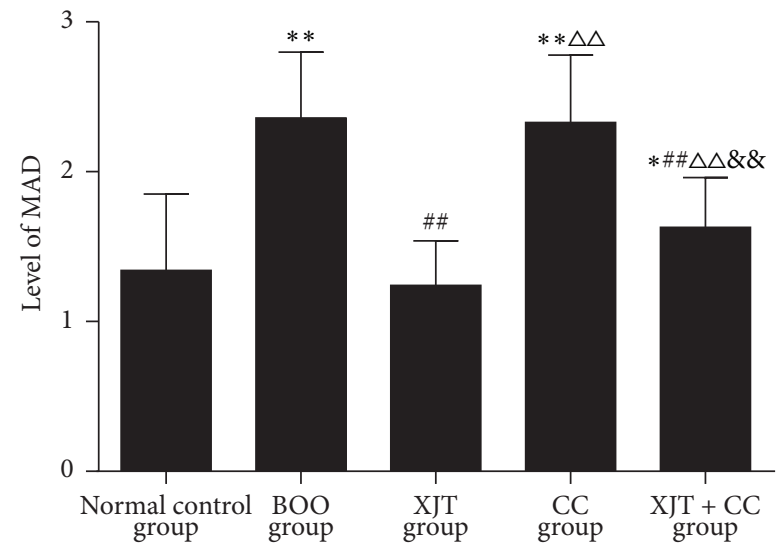

(a)

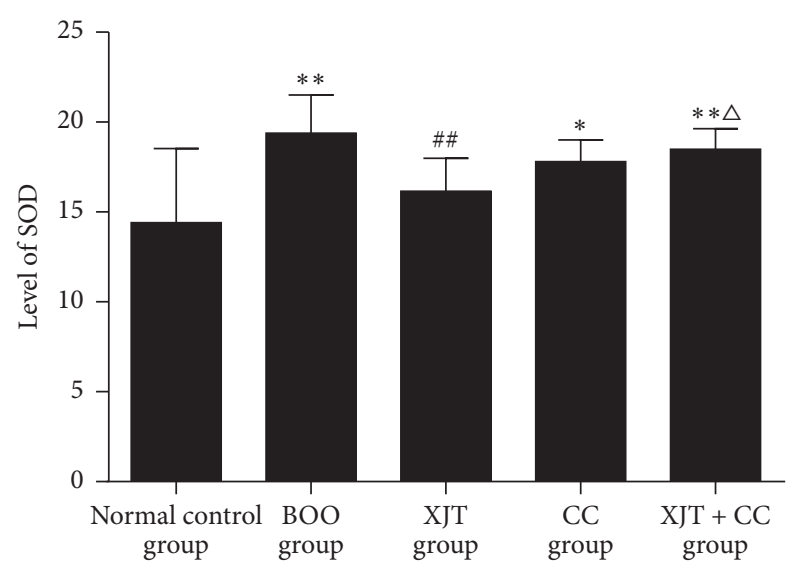

(b)

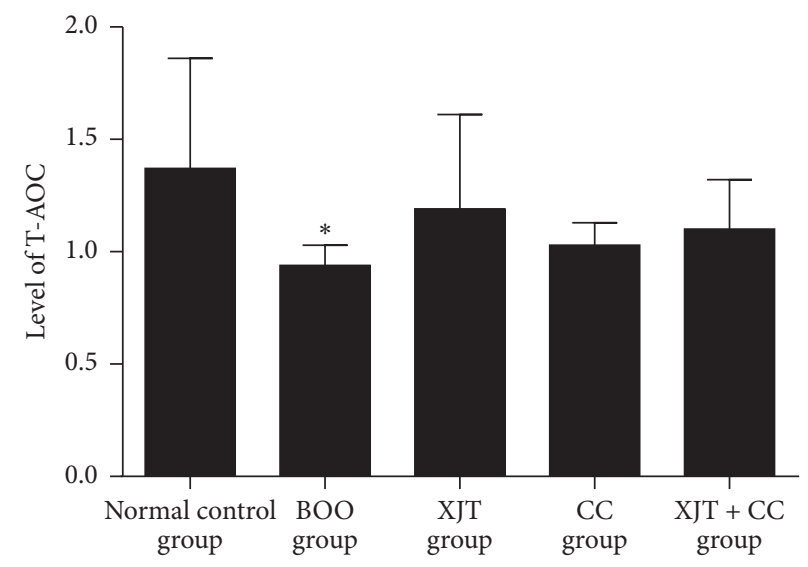

(c)

FIGURE 1: The levels of MAD (a), SOD (b), and T-AOC (c) in detrusor of rats in each group. BOO: bladder outlet obstruction; XJT: XianJia-Tang (a traditional Chinese medicine formula for invigorating kidney and promoting blood circulation); CC: Cesium Chloride; MAD: Malondialdehyde; SOD: superoxide dismutase; and T-AOC: total antioxidative capacity. * and $\triangle$, respectively, represent significant difference compared with normal control and XJT groups, with $P<0.05 ; * *$,\#, $\triangle \triangle$, and \&\&, respectively, represent significant difference compared with normal control, BOO, XJT, and CC groups, with $P<0.01$.

TABLE 4: The mRNA expression of potassium channels in detrusor of each group.

\begin{tabular}{|c|c|c|c|c|c|}
\hline Groups & $B K$ & $S K 2 / 3$ & $K v 2.1$ & $K v 1.5$ & KATP \\
\hline Normal control group & $13.38 \pm 1.41$ & $16.98 \pm 2.28$ & $38.20 \pm 8.23$ & $7.98 \pm 1.18$ & $8.88 \pm 1.59$ \\
\hline BOO group & $3.30 \pm 0.50^{* *}$ & $12.49 \pm 1.72^{* *}$ & $93.78 \pm 7.83^{* *}$ & $10.99 \pm 1.21^{* *}$ & $4.47 \pm 1.09^{* *}$ \\
\hline XJT group & $4.59 \pm 0.70^{* * \# \#}$ & $24.23 \pm 2.99^{* * \# \#}$ & $59.30 \pm 6.66^{* * \# \#}$ & $9.41 \pm 1.46^{* * \#}$ & $15.32 \pm 1.93^{* * \# \#}$ \\
\hline CC group & $2.66 \pm 0.34^{* * \Delta \Delta}$ & $12.68 \pm 2.64^{* * \Delta \triangle}$ & $74.23 \pm 14.30^{* * \# \triangle \triangle}$ & $11.37 \pm 1.57^{* * \triangle}$ & $9.19 \pm 1.19^{\# \# \triangle \triangle}$ \\
\hline XJT + CC group & $3.13 \pm 0.67^{* * \Delta}$ & $11.16 \pm 2.70^{* * \Delta \Delta}$ & $63.12 \pm 4.62^{* * \# \Delta \triangle \triangle 8}$ & $9.28 \pm 0.96^{* * \# \&}$ & $13.35 \pm 2.40^{* * \# \# \triangle \& \&}$ \\
\hline
\end{tabular}

BOO: bladder outlet obstruction; XJT: Xian-Jia-Tang (a traditional Chinese medicine formula for invigorating kidney and promoting blood circulation); and CC: Cesium Chloride. \#, $\triangle$, and \&, respectively, represent significant difference compared with BOO, XJT, and CC group with $P<0.05 ; * *$, \#\#, $\triangle \triangle$, and \&\&, respectively, represent significant difference compared with normal control, BOO, XJT, and CC group with $P<0.01$.

function. In addition, results also showed the effect of XJT on the oxidative stress level and potassium channels gene expressions in detrusor of $\mathrm{BOO}$ model rats. However, these effects could be significantly weakened by the injection of CC.

BOO could lead to the decrease of blood flow and oxygen tension in the detrusor layer, thereby generating an amount of reactive oxygen species $[17,18]$. Some studies have proved the efficacy of antioxidant therapy in the treatment of detrusor dysfunction in BOO animals $[19,20]$. In this study, although the effect of XJT on inhibiting the decrease of T-AOC was not statistically significant, the trend is visible. Besides, XJT significantly reduced the increase of MAD and SOD in BOO model rats, which illustrated XJT may protect the detrusor through inhibiting the oxidative stress. 


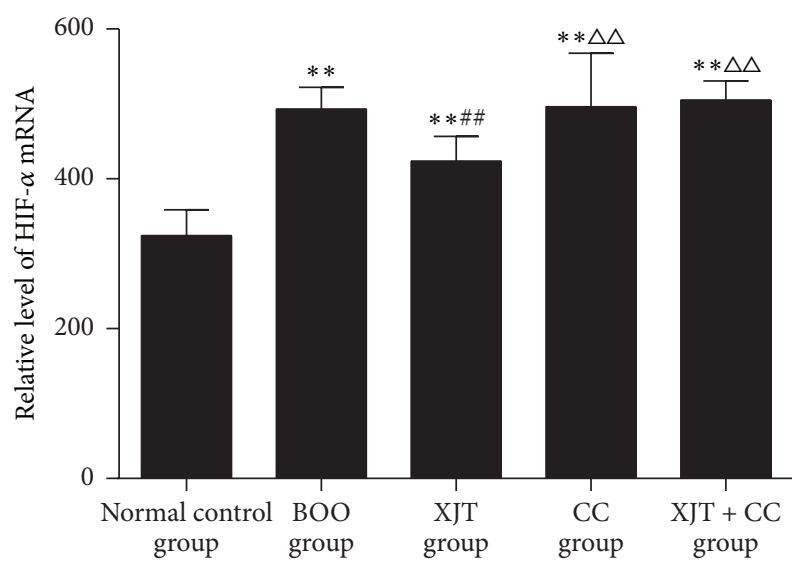

(a)

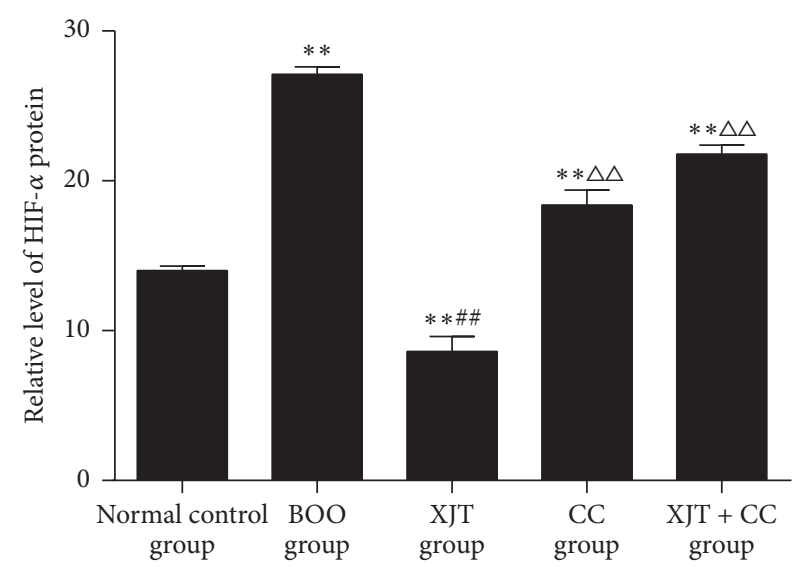

(b)

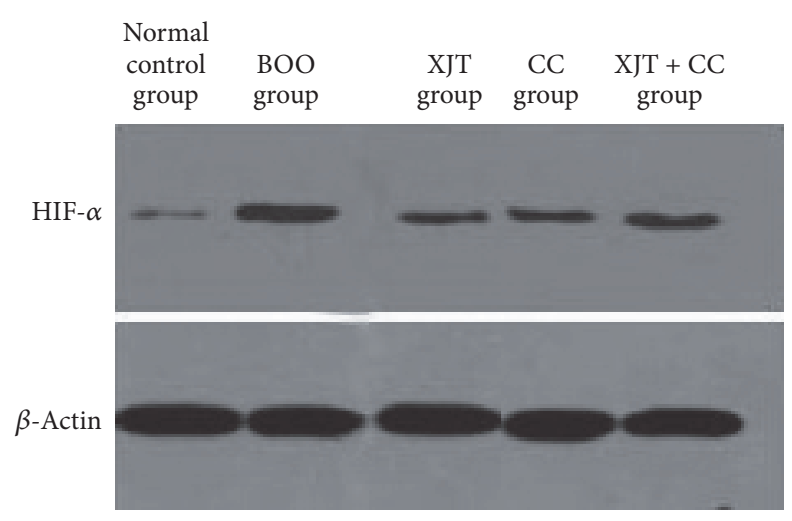

(c)

FIGURE 2: The relative expression of HIF- $\alpha$ in detrusor of rats in each group. BOO: bladder outlet obstruction; XJT: Xian-Jia-Tang (a traditional Chinese medicine formula for invigorating kidney and promoting blood circulation); CC: Cesium Chloride; and HIF- $\alpha$ : hypoxia inducible factor- $\alpha . * *$, \#\#, and $\triangle \triangle$, respectively, represent significant difference compared with normal control, BOO and XJT groups with $P<0.01$.

Potassium channels also play important roles in the stability and contractility of detrusor [21]. As reported, downregulation of $\mathrm{BK}$ channels may contribute to $\mathrm{BOO}$-induced detrusor overactivity by increasing the phosphorylation level of myosin light chain 2 [14]. Moreover, BK and SK channels were associated with the modulation of human detrusor smooth muscle phasic contractility [22]. The KATP channels were reported to mediate the contractions of detrusor muscles [23]. In this study, the anomalous expressions of potassium channels were found in $\mathrm{BOO}$ model rats. XJT significantly inhibited the expressions of $K v 2.1$ and $K v 1.5$ but increased the expression of $B K, S K 2 / 3$, and $K A T P$ in detrusor. These results indicated that the effect of XJT on BOO was associated with potassium channels in detrusor. Moreover, the overexpression of KATP was found in $\mathrm{BOO}$ model rats treated with XJT. In addition, results also showed that CC (a potassium channel inhibitor) could significantly weaken the effect of XJT on not only the expression of these potassium channels but also the bladder weight, urodynamics, and oxidative stress. It was reported that the KATP channels were associated with apoptosis induced by oxidative stress [24]. Moreover, the BOO-induced apoptosis in the urinary bladder has been found to be related to the hypoxia-triggered endoplasmic reticulum stress [25], which may be one of the reasons causing the oxidative stress in detrusor of BOO [26]. Thus, XJT may inhibit the oxidative stress induced injury through the activity of KATP channels in BOO model rats.

In addition, results also showed a significantly increased expression of HIF- $\alpha$ mRNA and protein in detrusor. Similarly, these increases were significantly inhibited by XJT and CC significantly affected this inhibition effect. As a nuclear transcription factor, HIF- $\alpha$ was always highly expressed in anoxic environment, which was an effective marker for hypoxia [27]. In BOO, high pressure in bladder wall always occurred, which could lead to reducing blood supply to the bladder due to oppressed artery [28]. Although subsequent process of urination can relieve the ischemia and hypoxia of bladder tissue, ischemia-reperfusion always enhances the bladder injury [29]. As shown in above discussion, the hypoxia may be the main reason resulting in the oxidative stress in detrusor of BOO [26, 30]. Previous studies have showed that KATP channels showed protective roles in hypoxia induced damages in nerve and vasculature [31, 32]. This evidence proved that KATP channels may play key roles in the inhibition effect of XJT on hypoxia and oxidative stress induced injury in detrusor. 


\section{Conclusions}

In conclusion, XJT was found to be effective in the treatment of BOO by inhibiting oxidative stress and increasing the expression of KATP channels in detrusor. The KATP channels may play key roles in protecting the detrusor from oxidative stress induced injury in the BOO model rats treated by XJT. The oxidative stress in detrusor may be caused by hypoxia. However, as a Chinese medicine formula, XJT was composed of a variety of drugs. The active ingredients in XJT and the interaction mechanisms among ingredients should be further explored in further studies.

\section{Acronyms}

BOO: Bladder outlet obstruction

LUTS: Lower urinary tract symptoms

BPH: Benign prostate hyperplasia

BK: $\quad$ Large-conductance calcium-and voltage-activated potassium channels

XJT: Xian-Jia-Tang

CC: $\quad$ Cesium Chloride

HIF- $\alpha$ : Hypoxia inducible factor- $\alpha$

MBC: $\quad$ Maximum bladder capacity

MDP: $\quad$ Maximum detrusor pressure

RT-PCR: Real-time polymerase chain reaction

MAD: Malondialdehyde

SOD: $\quad$ Superoxide dismutase

T-AOC: Total antioxidative capacity

KATP: Activates adenosine triphosphate-sensitive potassium.

\section{Conflicts of Interest}

The authors declare that there are no conflicts of interest.

\section{Authors' Contributions}

Jie Sun and Wei Shen contributed equally to this work.

\section{Acknowledgments}

All the authors would like to acknowledge the support from Zhejiang Provincial Natural Science Foundation Project. The study was supported by Zhejiang Provincial Natural Science Foundation Project (no. LY12H27005).

\section{References}

[1] J. J. M. C. H. De La Rosette, W. P. J. Witjes, W. Schäfer et al., "Relationships between lower urinary tract symptoms and bladder outlet obstruction: results from the ICS-'BPH' study," Neurourology and Urodynamics, vol. 17, no. 2, pp. 99-108, 1998.

[2] N. D. Patel and J. K. Parsons, "Epidemiology and etiology of benign prostatic hyperplasia and bladder outlet obstruction," Indian Journal of Urology, vol. 30, no. 2, pp. 170-176, 2014.

[3] D. P. J. Michielsen, T. Debacker, V. De Boe et al., "Bipolar transurethral resection in saline-an alternative surgical treatment for bladder outlet obstruction?" The Journal of Urology, vol. 178, no. 5, pp. 2035-2039, 2007.
[4] M. A. Collins, Transurethral Resection of the Prostate, Edited by E. D. Kim, Medscape, 2016.

[5] M. Muntener, S. Aellig, R. Kuettel, C. Gehrlach, T. Sulser, and R. T. Strebel, "Sexual function after transurethral resection of the prostate (TURP): results of an independent prospective multicentre assessment of outcome," European Urology, vol. 52, no. 2, pp. 510-516, 2007.

[6] J. Rassweiler, D. Teber, R. Kuntz, and R. Hofmann, "Complications of transurethral resection of the prostate (TURP)incidence, management, and prevention," European Urology, vol. 50, no. 5, pp. 969-980, 2006.

[7] G. Novara, A. Galfano, V. Ficarra, and W. Artibani, "Anticholinergic drugs in patients with bladder outlet obstruction and lower urinary tract symptoms: a systematic review," European Urology, vol. 50, no. 4, pp. 675-683, 2006.

[8] M. Creta, F. Bottone, S. Sannino et al., "Effects of alphal-blockers on urodynamic parameters of bladder outlet obstruction in patients with lower urinary tract symptoms suggestive of benign prostatic enlargement: a review," The Italian Journal of Urology and Nephrology, vol. 68, no. 2, 2015.

[9] A. Athanasopoulos, K. Gyftopoulos, K. Giannitsas, I. Fisfis, P. Perimenis, and G. Barbalias, "Combination treatment with an alpha-blocker plus an anticholinergic improves quality of life in patients with bladder outlet obstruction. A prospective, randomized, controlled study," Neurourology and Urodynamics, vol. 21, no. 4, p. 19, 2002.

[10] R. Radhakrishnan, N. Mohan, and A. Chawla, "Evaluation of the treatment pattern, clinical outcomes, and quality of life in patients with bladder outlet obstruction in a South Indian hospital," The Journal of Medicine Use in Developing Countries, vol. 1, no. 3, pp. 14-23, 2009.

[11] B. Çetinel and B. Onal, "Rationale for the use of anticholinergic agents in overactive bladder with regard to central nervous system and cardiovascular system side effects," Korean Journal of Urology, vol. 54, no. 12, pp. 806-815, 2013.

[12] S. A. Kaplan, "Side effects of $\alpha$-blocker use: retrograde ejaculation," Reviews in Urology, vol. 11, supplement 1, pp. S14-S18, 2009.

[13] J. G. Cao and A. F. Zhou, "Research on the effect of Xian Jia prescription restraining experimental benign prostatic hyperplasia rats," Chinese Journal of Experimental Traditional Medical Formulae, vol. 11, no. 1, pp. 43-45, 2005.

[14] S. Chang, C. M. Gomes, J. A. Hypolite et al., "Detrusor overactivity is associated with downregulation of large-conductance calcium- and voltage-activated potassium channel protein," American Journal of Physiology-Renal Physiology, vol. 298, no. 6, pp. F1416-F1423, 2010.

[15] M. Oelke, J. Baard, H. Wijkstra, J. J. de la Rosette, U. Jonas, and K. Höfner, "Age and bladder outlet obstruction are independently associated with detrusor overactivity in patients with benign prostatic hyperplasia," European Urology, vol. 54, no. 2, pp. 419-426, 2008.

[16] N. Iguchi, A. P. Malykhina, and D. T. Wilcox, "Inhibition of HIF reduces bladder hypertrophy and improves bladder function in murine model of partial bladder outlet obstruction," Journal of Urology, vol. 195, no. 4, pp. 1250-1256, 2016.

[17] J. E. Greenland, J. J. Hvistendahl, H. Andersen et al., "The effect of bladder outlet obstruction on tissue oxygen tension and blood flow in the pig bladder," BJU International, vol. 85, no. 9, pp. 1109-1114, 2000.

[18] A. T.-L. Lin, C. H. Yang, K.-K. Chen, and L. S. Chang, "Detrusor mitochondrial lipid peroxidation and superoxide dismutase 
activity in partial bladder outlet obstruction of rabbits," Neurourology and Urodynamics, vol. 24, no. 3, pp. 282-287, 2005.

[19] M. Oka, T. Fukui, M. Ueda, M. Tagaya, T. Oyama, and M. Tanaka, "Suppression of bladder oxidative stress and inflammation by a phytotherapeutic agent in a rat model of partial bladder outlet obstruction," Journal of Urology, vol. 182, no. 1, pp. 382390, 2009.

[20] M. H. Parekh, M. H. Parekh, R. Lobel et al., "Protective effect of vitamin $\mathrm{E}$ on the response of the rabbit bladder to partial outlet obstruction," Journal of Urology, vol. 166, no. 1, pp. 341346, 2001.

[21] G. V. Petkov, "Role of potassium ion channels in detrusor smooth muscle function and dysfunction," Nature Reviews Urology, vol. 9, no. 1, pp. 30-40, 2012.

[22] B. Darblade, D. Behr-Roussel, S. Oger et al., "Effects of potassium channel modulators on human detrusor smooth muscle myogenic phasic contractile activity: potential therapeutic targets for overactive bladder," Urology, vol. 68, no. 2, pp. 442-448, 2006.

[23] J. K. Badawi, R. Kirschner-Hermanns, and A. Ding, "Inhibitory effects of the ATP-sensitive potassium channel openers cromakalim, pinacidil and minoxidil on the carbachol-response curve in porcine detrusor muscle," Arab Journal of Urology, vol. 10, no. 2, pp. 207-215, 2012.

[24] Y. Teshima, M. Akao, W. A. Baumgartner, and E. Marbán, "Nicorandil prevents oxidative stress-induced apoptosis in neurons by activating mitochondrial ATP-sensitive potassium channels," Brain Research, vol. 990, no. 1-2, pp. 45-50, 2003.

[25] N. Sawada, J. Yao, N. Hiramatsu et al., "Involvement of hypoxiatriggered endoplasmic reticulum stress in outlet obstructioninduced apoptosis in the urinary bladder," Laboratory Investigation, vol. 88, no. 5, pp. 553-563, 2008.

[26] R. Inagi, "Endoplasmic reticulum stress as a target of therapy against oxidative stress and hypoxia," in Studies on Renal Disorders, pp. 657-672, Springer, Berlin, Germany, 2011.

[27] A. Zimna and M. Kurpisz, "Hypoxia-inducible factor-1 in physiological and pathophysiological angiogenesis: applications and therapies," BioMed Research International, vol. 2015, Article ID 549412, 13 pages, 2015.

[28] D. Xu, X. Cui, C. Qu, L. Yin, C. Wang, and J. Chen, "Urodynamic pattern distribution among aged male patients with lower urinary tract symptoms suggestive of bladder outlet obstruction," Urology, vol. 83, no. 3, pp. 563-569, 2014.

[29] A. Yildirim, F. F. Önol, G. Haklar, and T. Tarcan, "The role of free radicals and nitric oxide in the ischemia-reperfusion injury mediated by acute bladder outlet obstruction," International Urology and Nephrology, vol. 40, no. 1, pp. 71-77, 2008.

[30] R. Williams, P. Lemaire, P. Lewis et al., "Chronic intermittent hypoxia increases rat sternohyoid muscle NADPH oxidase expression with attendant modest oxidative stress," Frontiers in Physiology, vol. 6, article 15, 2015.

[31] L. Wang, Q.-L. Zhu, G.-Z. Wang et al., "The protective roles of mitochondrial ATP-sensitive potassium channels during hypoxia-ischemia-reperfusion in brain," Neuroscience Letters, vol. 491, no. 1, pp. 63-67, 2011.

[32] S. Bodiga, J. R. Falck, R. Zhang, Y. Gao, E. R. Jacobs, and M. M. Medhora, "Protection of mouse pulmonary arteries from hypoxia-induced apoptosis: cross talk between phosphoinositide 3-kinase (PI3K) and ATP-sensitive potassium (KATP) channels," The FASEB Journal, vol. 22, no. 1, meeting abstracts 915.2, 2008 . 


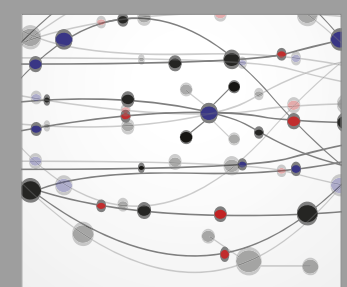

The Scientific World Journal
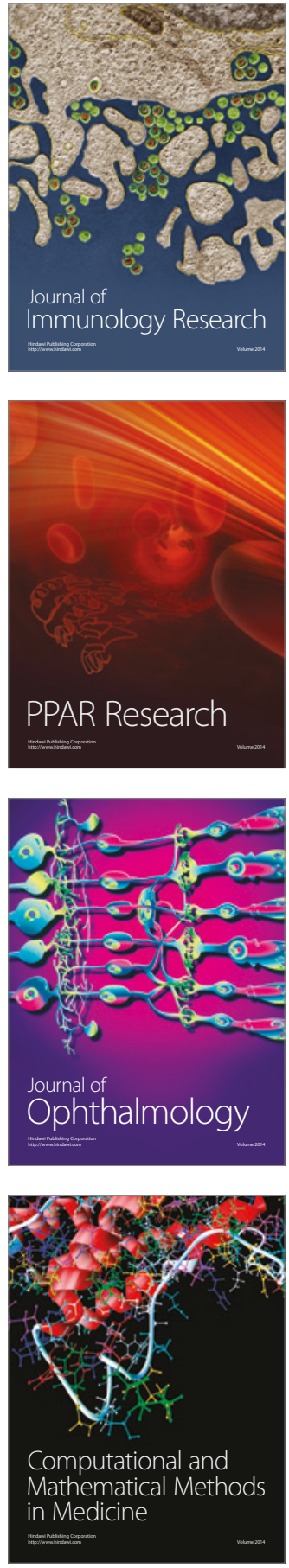

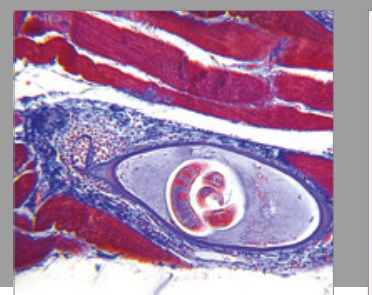

Gastroenterology Research and Practice
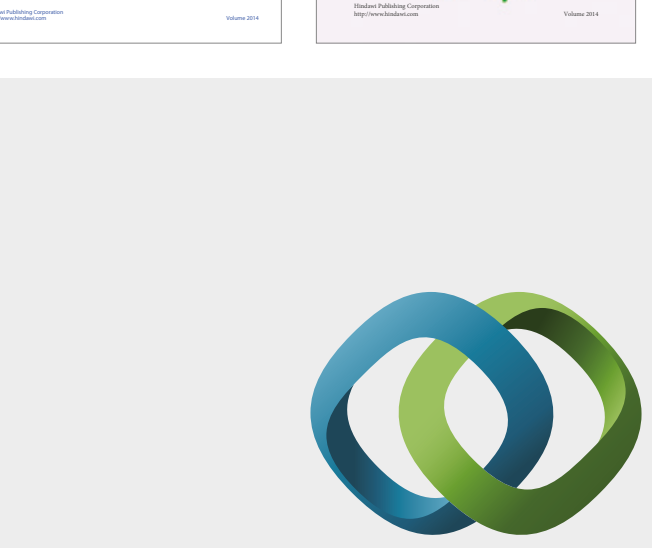

\section{Hindawi}

Submit your manuscripts at

https://www.hindawi.com
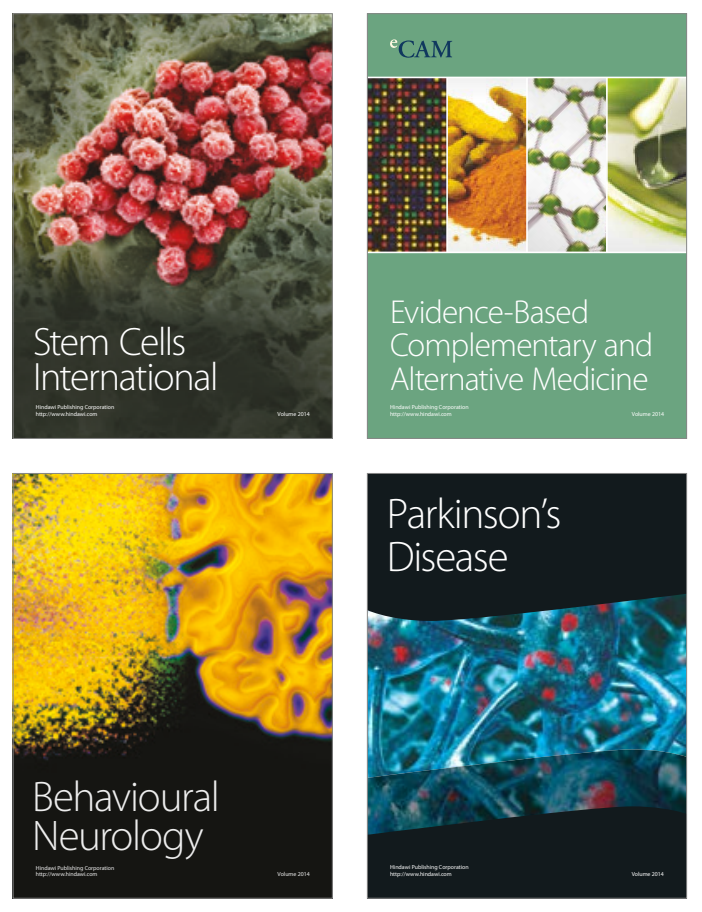
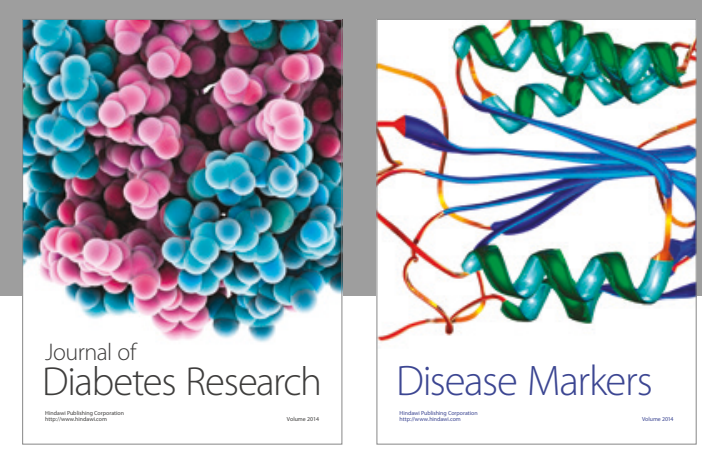

Disease Markers
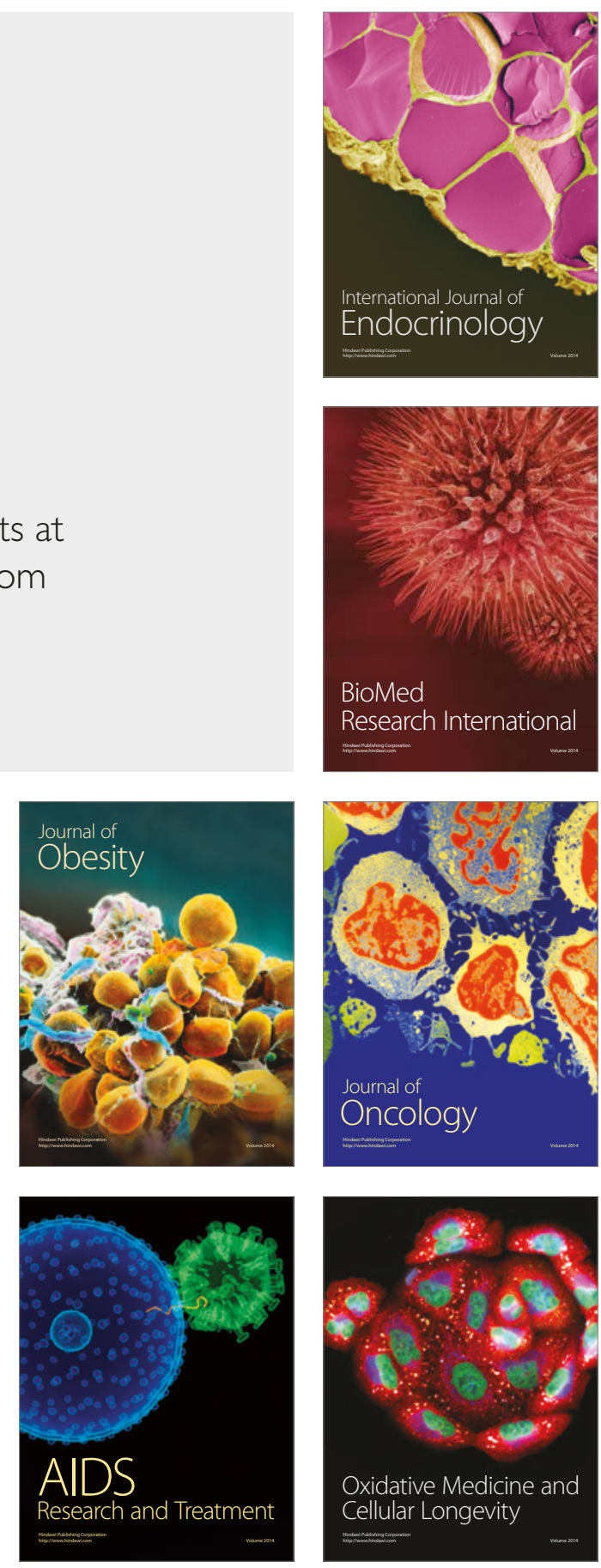